\title{
Hawking Radiation and Analogue Experiments: A Bayesian Analysis
}

\author{
Radin Dardashti $^{* 1}$, Stephan Hartmann ${ }^{\dagger 2}$, Karim Thébault ${ }^{\ddagger 3}$, and \\ Eric Winsberg ${ }^{\S 4}$ \\ ${ }^{1}$ Interdisziplinäres Zentrum für Wissenschafts- und Technikforschung/ Philosophisches Seminar, \\ Bergische Universität Wuppertal \\ ${ }^{2}$ Munich Center for Mathematical Philosophy, Ludwig Maximilians Universität München \\ ${ }^{3}$ Department of Philosophy, University of Bristol \\ ${ }^{4}$ Department of Philosophy, University of South Florida
}

June 25, 2018

\begin{abstract}
We present a Bayesian analysis of the epistemology of analogue experiments with particular reference to Hawking radiation. First we prove such experiments can be confirmatory in Bayesian terms based upon appeal to 'universality arguments'. We then provide a formal model for the scaling behaviour of the confirmation measure for multiple distinct realisations of the analogue system and isolate a generic saturation feature. Finally, we demonstrate that different potential analogue realisations could provide different levels of confirmation. Our results provide a basis both to formalise the epistemic value of analogue experiments that have been conducted and to advise scientists as to the respective epistemic value of future analogue experiments.
\end{abstract}

\footnotetext{
*dardashti@uni-wuppertal.de

†'s.hartmann@lmu.de

${ }_{\ddagger}^{\ddagger}$ karim.thebault@bristol.ac.uk

§winsberg@usf.edu
} 


\section{Contents}

1 Introduction 2

2 Confirmation, Analogy and Experiment 3

3 Hawking Radiation and Universality 6

4 Bayesian Analysis 9

4.1 Single Source Confirmation . . . . . . . . . . . . . . . . . . 9

4.2 Multiple Source Confirmation . . . . . . . . . . . . . . . . . . 14

4.3 Confirmation Dependence on Source System . . . . . . . . . . . . . . 16

5 Conclusion and Prospectus 17

$\begin{array}{lll}\text { A Proof of Theorem } 1 & 17\end{array}$

$\begin{array}{llr}\text { B Proofs for } n \text { Source Systems } & 18\end{array}$

C Proof of Theorem $3 \quad 20$

\section{Introduction}

In a landmark paper, Jeff Steinhauer (2016) reported the experimental observation of Hawking radiation in an acoustic analogue black hole (or 'dumb hole') constructed using a Bose-Einstein Condensate (BEC). ${ }^{1}$ This remarkable result has met with a strangely divergent response in the physics community, varying from the assertion that it provides 'experimental confirmation of Hawking's prediction' (Jeff Steinhauer quoted in Haaterz), to dismissal simply as 'an amusing feat of engineering' that 'won't teach us anything about black holes' (Daniel Harlow quoted in Quanta). What can we learn from such analogue black hole experiments? Are there circumstances in which they can be taken to provide inductive support for conclusions about astrophysical black holes?

In this paper we will substantially extend previous philosophical work characterising analogue black hole experiments as a form of 'analogue simulation' (Dardashti et al. 2017; Thébault 2016) via application of Bayesian confirmation theory. In that previous analysis, emphasis was placed upon the qualitative claim that certain 'universality arguments' can be used to link evidence about the 'source' dumb hole system to the 'target' black hole system. The results of this paper are quantitive in nature and licence normatively valuable conclusions regarding the structure of such inferences.

We first present a theorem that demonstrates how the confirmation claim can be qualitatively characterised in Bayesian terms. The role of the universality arguments is understood in terms of support for background assumptions that are common between the source and target models. This means that there is a binary variable

\footnotetext{
${ }^{1}$ We should note that the Steinhauer result is novel in that it is the first clear experimental demonstration of quantum Hawking radiation. The earlier experiment of Weinfurtner, Tedford, Penrice, Unruh, and Lawrenc (2013), using surface water waves and an analogue white hole, showed 'classical aspects' of Hawking radiation. See also (Euvé, Robertson, James, Fabbri, and Rousseaux 2018).
} 
that can be assumed to be positively correlated with the empirical adequacy of both the source and target models. Evidence in favour of the model of the source system can thus be used to make inferences about the target system. Although not in-andof-itself a validation of the argument for confirmation via analogue simulation, the Bayesian analysis demonstrates the internal consistency of the informal arguments given in (Dardashti et al. 2017). Furthermore, the formal model provides a powerful evaluative and heuristic tool for the further analysis of the structure of the inferences involved in cases of analogue simulation. In particular, in the remains of the paper, we present two further results that we expect will be of interest to contemporary analogue black hole experimentalists.

Our second principal result relates to the behaviour of the confirmation measure in the context of multiple 'materially distinct' experimental realisations of the analogue system. This is of particular relevance to contemporary analogue black hole research since the BEC experiment is expected to be the first of many diverse analogue realisations of Hawking radiation: e.g., phonons in superfluid liquid helium or 'slow light' in moving optical media. The immediate question in this context is how many distinct analogue systems one needs to construct to be confident in the astrophysical effect. The second key result of this paper is a formal model for 'multiple source' analogue simulation displaying the generic feature of 'saturation' in confirmatory power with an increase in the number of sources.

Finally, and perhaps most intriguingly, there is the question of whether different potential analogue realisations could provide different levels of confirmation. Would we learn more about astrophysical black holes from an analogue experiment based upon liquid helium or optical media? The third key result of the paper is a theorem proving that analogue experiments in which we are more confident about the fundamental physics (e.g. optical media) teach us less about the target system than those about which we are less confident (e.g. liquid helium). Our results thus provide a basis to both formalise the epistemic value of analogue experiments that have been conducted, like that of Steinhauer, and to advise scientists as to the respective epistemic value of future analogue experiments. As such, our work demonstrates the enduring value of the Bayesian framework as a tool for analysing the protean forms of scientific inference.

\section{Confirmation, Analogy and Experiment}

The literature on analogical reasoning in science is fairly extensive, with particularly noteworthy contributions by Keynes (1921), Hesse (1963, 1964, 1974), Bailer-Jones (2009) and Bartha $(2010,2013){ }^{2}$ Typically analogical arguments have the form of abstract speculative inferences regarding possible features of one system ('the target') based on known features of another system ('the source'). Classic examples are Reid's argument for the existence of life on other planets based upon life on earth (Reid and Hamilton 1850) or Hume's argument for animal consciousness based upon human consciousness (Hume 1978).

\footnotetext{
${ }^{2}$ See also Norton (2011) for an importantly different take on analogical arguments. Norton's analysis focuses on analogical arguments that proceed via subsumption of the target system into a larger class of entities, including the source system. There are broad parallels between the structure of such inferences and our analysis.
} 
Analogical arguments evidently play an important heuristic role in scientific practice in that they provide 'cognitive strategies for creative discovery' (Bailer-Jones 2009, p. 56). The epistemic role of analogical arguments in science is, however, more controversial. In particular, the literature contains diverging answers regarding whether analogical arguments can provide Bayesian confirmation of a hypothesis regarding the target system. From a Bayesian perspective on confirmation, evidence for a hypothesis can count as confirmatory only if the probability of the hypothesis given the evidence together with certain background assumptions is larger than the probability of the hypothesis given only the background assumptions. In a detailed and nuanced treatment of the issue, Hesse (1974, pp. 208-19) suggests that analogical arguments can in some cases be confirmatory in a Bayesian sense, so long as the analogical relationship that holds is in terms of what she calls a 'material analogy'. That is, where there is a similarity relation of sharing at least one predicate between the target and source systems. ${ }^{3}$

Contrastingly, Bartha $(2010,2013)$ offers arguments that analogical arguments cannot in principle be confirmatory in a Bayesian sense. In particular, he suggest that because the information encapsulated in an analogical argument is taken to already be part of the 'background knowledge', the probability of a hypothesis regarding the target system must be identical before and after including the analogical argument. Bartha takes this instance of the familiar problem of old evidence (Glymour 1980) to be significant enough to bar analogical arguments from being confirmatory in Bayesian terms. Rather, following Salmon $(1967,1990)$, Bartha argues that arguments by analogy can establish only the plausibility of a conclusion in the technical sense of justifying the assignment of a non-negligible prior probability assignment (Bartha $2010, \S 8.5)$. On this analysis, it is not in principle possible for analogical arguments to confer inductive support for a hypothesis. That is, although analogical arguments can certainly be stronger or weaker, even the strongest possible analogical argument cannot confer confirmation in a Bayesian sense: they are abstract inferences that can only ever support plausibility claims rather than providing inductive evidence.

Although worthy and insightful, the treatments of Hesse (1974) and Bartha (2010) do not extend to the analysis of analogue experiments. This is because analogue experiments are unlike arguments by analogy in exactly the respects that are crucial for either the Hesse or the Bartha analysis to go through. The question of whether one agrees with Hesse or Bartha about the confirmatory power of arguments by analogy is simply tangential to the analysis of the confirmatory power of analogue experiments. In the case of Hesse, this is indicated by the fact that her notion of material analogy is too strict to accommodate the subtle relation that the model of the target has to the model of the source in the case of analogue experiments, such as the Steinhauer experiments. We are dealing with an analogue simulation that does not involve a material analogy in the sense of Hesse since there is not a physical property common between the target and source systems. Rather than a material relation between systems, we have a syntactic isomorphism between models. ${ }^{4,5}$

\footnotetext{
${ }^{3}$ Hesse (1974, p. 216) explicitly rules out the possibility of confirmation obtaining in cases where there is purely a 'formal analogy'. That is, where target and source are both interpretations of the same formal calculus but do not share material similarities.

${ }^{4}$ Here 'syntactic isomorphism' is a natural generalisation of Hempel's (1965) notion of nomic isomorphism to the case of a relation between models rather than laws.

${ }^{5}$ Hesse (1963), does in fact, rather presciently, consider the relevance of simulators in her account
} 
Bartha's negative analysis of the prospect for confirmation via analogical argument is similarly inapplicable to analogue experiments. In this case because of the 'experimental' rather than the 'simulation' side of things. Analogue experiments, unlike analogical arguments, are essentially empirical: they involve learning about the world by manipulating it. In experiments, such as those carried out by Steinhauer, we manipulate the source such that certain explicit modelling assumptions matching those for the target obtain. Analogue simulation thus resembles a form of experimentation, involving the 'programming' of a physical system such that it can be used to 'simulate' another physical system. Thus, we see that conclusions from the philosophical analysis of analogical argument should not be taken to be readily extendible to cases of analogue simulation in contemporary science. In particular, it is self-evidently the case that the old evidence problem for the Bayesian analysis of traditional arguments by analogy à la Hesse, is not longer relevant for analogue experiments. Analogue experiments unlike analogical reasoning explicitly involve the collection of new evidence and there are not good grounds for relegating their significance to mere plausibility.

This collection of new evidence motivates us to consider the 'epistemology of analogue experimentation' in parallel with the epistemology of conventional experiments. As has been noted by various authors (Franklin 1989; Winsberg 2010; Franklin and Perovic 2016)) conventional experiments are generally only of epistemological significance when supplemented by arguments that imply that the information learned about the system being manipulated ('the source') is relevantly probative about the class of systems that are of interest to the experimenters ('the target'). A nice illustration of this point is provided by experiments designed to learn about the thermal conductivity of the iron in Earth's core (Konôpková et al. 2016; Dobson 2016). The experiments were carried out in the lab using samples of iron that are placed in a laser-heated diamond-anvil cell. The pressure and temperature that the iron samples were subjected to were specifically matched to those relevant to the cores of Mercurysized to Earth-sized planets. For the experiments to achieve their epistemic purpose, they must be supplemented with arguments that the measurements are be 'relevantly probative' of the thermal conductivity of iron in the core's of Mercury-sized to Earthsized planets. That is, there must be a basis to generalise from the observations regarding the lab based system (the 'source'), to relevant systems outside the lab (the 'target'). In parallel, what we take to be the key question in the epistemology of analogue experimentation is whether we can provide arguments that the relevant source systems 'stand-in' for the target systems to which the analogical relationship refers. Can we find arguments that the Steinhauer experiments are relevantly probative of the thermal properties of astrophysical black holes? ${ }^{6}$

of models and analogies in science. Tantalisingly, she says that analogue machines (i.e. simulators): 'are useful and necessary as predictive models precisely in those cases where the material substance of parts of the analogue is not essential to the model, but where the mutual relations of the parts are essential' (p. 102) This connection is unfortunately not taken up in the 1974 Bayesian analysis.

${ }^{6}$ See (Thébault 2016) for an analysis of the connection between the epistemology of analogue experiments and the notions of internal' and external validation as discussed in the philosophy of experimentation. 


\section{Hawking Radiation and Universality}

Hawking radiation (Hawking 1975) is a thermal phenomena that is predicted to be generically associated with black holes. Despite the absence of either a clear causal process behind the radiation or experimental evidence, it is widely believed in by theoretical physicists. In fact, the ability to recover Hawking radiation could even be taken as a theoretical test of prospective theories of quantum gravity, much like the recovery of the Bekenstein-Hawking formula for black hole entropy (Wüthrich 2017). There are two connected reasons why physicists are so confident in the prediction of Hawking radiation. First, given the Unruh effect (Unruh 1976), which associates a temperature with acceleration, Hawking radiation seems to be directly implied by the equivalence principle. ${ }^{7}$ Second, starting from Hawking's original calculation a remarkable number of different derivations of the effect have been given. ${ }^{8}$ The consensus is that the effect is 'remarkably robust' to the addition of complicating factors to the original derivation. The overall implication is that very general theoretical constraints coming from quantum field theory and general relativity (two well tested theories) necessitate that something like Hawking radiation must exist. The purpose of this paper is not to address the evidential import of such theoretical considerations. Rather, our focus is on the potential for analogue experiments to provide confirmatory evidence of a form akin to conventional experiments. This notwithstanding, questions of robustness will return to the fore in the context of a particular form of universality argument that will be found to be central for questions of confirmation. Before then, it will be instructive to consider the basic elements of the original Hawking derivation of a radiative flux for astrophysical black holes in comparison with their sonic analogues.

Hawking's analysis is performed in the context of a semi-classical approach to gravity. That is, we consider matter as described by quantum field theory and spacetime as described by a continuous classical geometry. Crucially, although the spacetime in question can have non-trivial curvature, it is not coupled to the quantum field. That is, there can be no 'back-reaction' between the quantum matter and classical geometry. For this modelling framework to be valid it is assumed that we are considering quanta of wavelengths much larger than the Planck length. Quanta of the order of the Planck length could be expected to 'see' the (presumed) non-classical and non-continuous structure of spacetime and would necessitate a quantum theory of gravity in their description. Quite general formal considerations can be used to show that in the semi-classical framework the vacuum state of a quantum scalar field defined at past null infinity need not appear as a vacuum state to observers at positive null infinity. In particular, it may contain a 'particle flux'. What Hawking shows in his original paper is that for spacetime in which an astrophysical black hole forms there will be a particle flux which observers at positive null infinity will associate with the blackhole horizon. The asymptotic form of the expression for the particles flux is shown to depend only upon the surface gravity of the black hole denoted by $\kappa_{G}$. Surface gravity is essentially the force per unit mass that must be applied at infinity in order to hold a stationary zero angular momentum particle just outside the horizon (Jacobson 1996). Hawking's calculation implies that a black hole has intrinsic

\footnotetext{
${ }^{7}$ Such a conclusion is, in fact, a little too quick since the equivalence principle holds only locally and Hawking radiation is a global effect. See (Helfer 2010).

${ }^{8}$ See (Leonhardt and Philbin 2008; Thompson and Ford 2008; Wallace 2017).
} 
properties that are connected to a non-zero thermal particle flux at late times. The precise relation takes the form:

$$
\left\langle\hat{N}_{\omega}\right\rangle=\frac{1}{e^{\frac{2 \pi \omega}{\hbar \kappa_{G}}}-1} \quad \text { with } \quad T_{B H}:=\hbar \kappa_{G} / 2 \pi,
$$

where $\hat{N}_{\omega}$ is the number operator for modes detected at late times with frequency $\omega$ and $\hbar$ is Planck's constant divided by $2 \pi$.

One key feature of the derivation of the temperature is worth noting here since it will be very important in what follows. In the derivation of Hawking radiation an exponential gravitational red-shift means that the black hole radiation detected at late times must be taken to correspond to extremely high frequency radiation at the horizon. These 'trans-Planckian' modes are of wavelengths that are of precisely the kind that we presumed to exclude in using the semi-classical framework. There is thus a tension between the initial modelling assumptions and the details of the calculation. We will return to this issue shortly.

Not long after the derivation of Hawking's radiation, it was proposed by Unruh that a similar thermal effect might exist in the context of sound in fluid systems (Unruh 1981). In particular, Unruh showed that the key elements of Hawking's calculation could be re-applied in the context of a semi-classical model of sound in fluids. The role of the spacetime geometry is now played by a 'bulk' fluid flow as described by continuum hydrodynamics. The role of the quantum field is then played by a quantized linear fluctuation within the fluid, a phonon. The modelling framework of continuum hydrodynamics is only valid provided fluid density fluctuations of the order of molecular lengths can be ignored. So for this semi-classical description to be adequate the wavelengths of the phonons must be much larger than the intermolecular distances. Unruh's brilliant insight was to recognise that there is a special class of analogue fluid systems for which the equations of semi-classical continuum hydrodynamics take a form isomorphic to those of semi-classical gravity. The role of the black hole event horizon is now played by an effective acoustic horizon where the inward flowing magnitude of the radial velocity of the fluid exceeds the speed of sound. The black hole is replaced by a dumb hole. Just as in the gravitational Hawking effect a black hole event horizon is associated with a late time thermal photonic flux, in the hydrodynamic Hawking effect Unruh showed that a dumb hole sonic horizon can be associated with a late time thermal phononic flux.

In practice, detection of Hawking radiation in a fluid is extremely difficult and it has not been possible to construct an acoustic analogue black hole of the type originally proposed by Unruh. Rather, an alternative medium for experimentally realisable acoustic blackholes was proposed in terms of Bose-Einstein condensates (Garay et al. 2000). This is an exotic form of matter predicted in the 1920s (Einstein 1924; Bose 1924) but not created experimentally until 1995 (Anderson et al. 1995). Crucially, in the limit of weak coupling and no backreaction density fluctuations in a BEC are described by the Gross-Pitaevskii equation. When variations in the density of the BEC occur on length scales much greater than the healing length, the Gross-Pitaevskii equation can be used to derive an equation of the same form as that of an irrotational fluid derived by continuum hydrodynamics. Following the same line of reasoning as Unruh's original ideal fluid argument, (Garay et al. 2000) derived a BEC Hawking Effect using appeal to this hydrodynamic approximation to a BEC. 
There are now a huge number of potential analogue realisations of the Hawking effect: phonons in superfluid liquid helium, 'slow light' in moving media, traveling refractive index interfaces in nonlinear optical media, laser pulses in nonlinear dielectric medium. ${ }^{9}$ To realize the Hawking effect it seems it is sufficient to have: i) a classical (effective) background with quantum fields living on it; and ii) an (effective) geometry with an (effective) causal horizon.

A crucial feature in the derivation of all these effects is the integration over extremely high energy 'trans-Planckian' modes. As noted above, in the black hole case these modes get included in the calculation due to an exponential redshift between the horizon (where they originate) and late times (where they are detected). Such a feature is generic to all models of Hawking radiation in which the modes originate near the horizon. ${ }^{10}$ Since the 'trans-Planckian' regime beyond the domain of applicability of the semi-classical modelling framework we are using, this problem of exponential redshift seems rather worrying. In fact, according to some, the transPlanckian problem is so serious as to cast doubt upon the Hawking calculation entirely. Unruh, for instance, even asks that if the derivation relies on such absurd physical assumptions, can the result be trusted?' (Unruh 2014, p. 534). The problem with 'trans-Planckian' modes has a direct analogue in both the continuum hydrodynamic and BEC derivations. In particular, the acoustic analogue of the gravitational redshift, means that in both cases we are including in our calculation phonons of wavelengths small enough to probe the regimes well beyond the inter-molecular length and healing length respectively.

Fortunately, there are good reasons to expect that the Hawking effect in both gravitational and analogue cases will be robust to disturbance from trans-Planckian modes. In particular, (Unruh and Schützhold 2005) have provided strong theoretical reasons to expect that, under certain conditions, any possible modifications to the Hawking flux by trans-Planckian modes will be negligible. ${ }^{11}$ Unruh and Schützhold show that a wide family of trans-Planckian effects can be factored into the calculation of Hawking radiation via a non-trivial dispersion relation. To lowest order and given certain modelling assumptions, Hawking radiation, both astrophysical and acoustic, is independent of the details of the underlying physics.

Following the influential account of (Batterman 2000), we can isolate two characteristic features of a universality argument: i) Many different system types, with physically distinct microstructure (e.g. fluids and magnets), exhibit the same behaviour; ii) Details of microstructure of a given token system are largely irrelevant for describing behaviour generically exhibited by members of the system type. Given this definition, we can plausibly take the work of Unruh and Schützhold to serve as a model for any theoretical argument towards the universality of the Hawking effect in the sense that it gives conditions under which: i) Many different realisations of the (analogue) black hole system, with distinct 'trans-Planckian' structure (e.g. black holes and BECs), exhibit the Hawking effect; and ii) Details of 'trans-Planckian' struc-

\footnotetext{
${ }^{9}$ See (Philbin et al. 2008; Belgiorno et al. 2010; Unruh and Schützhold 2012; Liberati et al. 2012; Nguyen et al. 2015; Jacquet 2018).

${ }^{10}$ It is worth noting here that whilst, the non-standard derivation of (Giddings 2016) does appear to allow one to avoid this feature, that of (Polchinski 1995), prima facie, does not (Harlow 2016, pp. 37-8).

${ }^{11}$ For further work on these issues, using a range of different methodologies, see for example (Corley 1998; Himemoto and Tanaka 2000; Barceló, Garay, and Jannes 2009; Coutant, Parentani, and Finazzi 2012). For philosophical discussion see (Dardashti, Thébault, and Winsberg 2017) and (Gryb, Palacios, and Thébault 2018).
} 
ture of a given (analogue) black hole system are largely irrelevant for describing the associated thermal behaviour.

There are thus plausible grounds to take the Unruh and Schützhold arguments to refer to universality in broadly the same sense as Batterman. Nothing, however, in our analysis will hang upon the strength of the connection between the two uses of the word universality. That is, whether or not one thinks the Unruh and Schützhold arguments establish the Hawking effect to be a 'universal phenomena', these arguments can still be unambiguously understood as having the three following implications. First, that there is a phenomenon (i.e., the thermal flux characteristic of Hawking radiation) that is found in systems that are substantially different in their microstructure but share crucial formal features (i.e., can be modelled using a quantum fluctuation propagating on a geometry with a causal horizon). Second, this phenomenon is robust to a wide class of variations in microstructure of a particular realisation (irrelevance of token details). Third, this phenomenon is robust to a wide class of variations in microstructure between different realisations (irrelevance of type details).

Together these three features comprise the precise sense of what we will mean by a 'universality argument for Hawking radiation'. Whilst detailed analysis of the particular universality argument of Unruh and Schützhold, is an important task, we will not further pursue it here. Rather, in the reminder of this paper, we will assume the position of an agent with significant credence in some universality arguments for Hawking radiation, and pursue the consequences for the epistemology of analogue experimentation.

\section{Bayesian Analysis}

\subsection{Single Source Confirmation}

The key claim that we wish to investigate is the sense in which analogue 'dumb hole' experiments can provide inductive support for a hypothesis regarding black holes given we believe the appropriate universality arguments. In what follows we give a Bayesian network representation of the proposed inferential structure of analogue simulation defended in (Dardashti et al. 2017) and show that the evidence in the source system can provide confirmation of hypotheses regarding the target system in certain circumstances. ${ }^{12}$

Let us start with the representation of the target system $\mathcal{T}$. We denote by $M$ a propositional variable that takes the two values:

M: The modelling framework $\mathcal{M}$ provides an empirically adequate description of the target system $\mathcal{T}$ within a certain domain of conditions $D_{\mathcal{M}}$.

$\neg \mathrm{M}$ : The modelling framework $\mathcal{M}$ does not provide an empirically adequate description of the target system $\mathcal{T}$ within a certain domain of conditions $D_{\mathcal{M}}$.

\footnotetext{
${ }^{12}$ For models of confirmation in terms of the Bayesian framework see (Hartmann and Sprenger 2010; Bovens and Hartmann 2004) or for the hypothetic-deductive framework see (Betz 2013). Throughout this paper, we follow the convention that propositional variables are printed in italic script, and that the instantiations of these variables are printed in roman script.
} 
The adequacy of the modelling framework $\mathcal{T}$ depends on whether the background assumptions which justify the empirical adequacy of the modelling framework obtain. We denote with $X_{M}$ the random variable with the values:

$\mathrm{X}_{M}$ : The background assumptions $x_{M}=\left\{x_{M}^{1}, x_{M}^{2}, \ldots, x_{M}^{n}\right\}$ are satisfied for system $\mathcal{T}$.

$\neg \mathrm{X}_{M}$ : The background assumptions $x_{M}=\left\{x_{M}^{1}, x_{M}^{2}, \ldots, x_{M}^{n}\right\}$ are not satisfied for system $\mathcal{T}$.

where the second statement should be read in terms of a negation of the disjuncts. The role of the background assumptions is to define and justify the domain of conditions for the model. These assumptions involve knowledge, both theoretical and empirical, that goes beyond what is encoded within the model. Such knowledge need not be in the form of a simple, unified framework. Rather the background knowledge of the people who build and use models can contain an incompletely integrated set of explicit and tacit ideas about when a particular modelling framework will be adequate for a particular purpose and to a particular desired degree of accuracy.

With this in mind, we can introduce the random variables $A$ and $X_{A}$ for the source system $\mathcal{S}$. Where $A$ is a propositional variable that takes the two values:

A: The modelling framework $\mathcal{A}$ provides an empirically adequate description of the source system $\mathcal{S}$ within a certain domain of conditions $D_{\mathcal{S}}$.

$\neg$ A: The modelling framework $\mathcal{A}$ does not provide an empirically adequate description of the source system $\mathcal{S}$ within a certain domain of conditions $D_{A}$.

and $X_{A}$ is the random variable with the values:

$\mathrm{X}_{A}$ : The background assumptions $x_{A}=\left\{x_{A}^{1}, x_{A}^{2}, \ldots, x_{A}^{k}\right\}$ are satisfied for system $\mathcal{S}$.

$\neg \mathrm{X}_{A}$ : The background assumptions $x_{A}=\left\{x_{A}^{1}, x_{A}^{2}, \ldots, x_{A}^{k}\right\}$ are not satisfied for system $\mathcal{S}$.

The systems $\mathcal{T}$ and $\mathcal{S}$ are assumed to differ in terms of their material constitution and the fundamental laws governing their dynamics. This means that the background assumptions behind the models $\mathcal{M}$ and $\mathcal{A}$ can reasonably be assumed to be very different. Given this, it is justified, prima facie, to assume that $X_{M}$ and $X_{A}$ are probabilistically independent. Furthermore, we have assumed that the source system is empirically accessible meaning we can gain empirical evidence regarding (at least) some of its consequences. We can encode this by introducing a variable $E$ corresponding to the two values, $E$, the empirical evidence obtains, and $\neg \mathrm{E}$, the empirical evidence does not obtain.

We can represent all the variables introduced thus far as well as the probabilistic dependencies using a Bayesian network (Bovens and Hartmann 2004). The random variables are represented as 'nodes' in the network (i.e. circles) and the probabilistic dependences as directed edges (i.e. arrows). We draw an arrow between two nodes when the variable in the 'parent node' has a direct influence on the variable in the 'child node'. Probabilistic independence is represented implicit by the absence of an arrow between two nodes. The entire set up thus far is represented by the Bayesian network in Figure 1. 


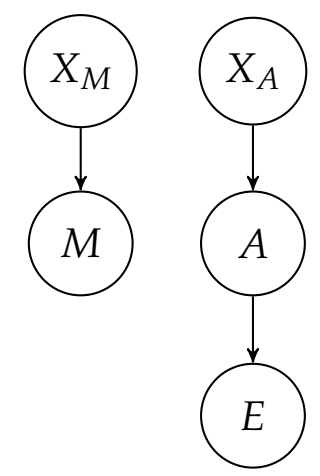

Figure 1: One source system without universality.

Consider the case of an analogue black hole experiment, leading an agent to believe $\mathrm{E}$, in a context where the agent does not believe in any form of universality argument. The equations of the source model are syntactically isomorphic to the equations that are supposed to govern the behaviour of the target model. This is somewhat surprising, given that one arrives at them from different starting points (semi-classical gravity vs. e.g. semi-classical continuum hydrodynamics) and by making different background assumptions. However, the agent has no reason to believe that the isomorphism represents a deeper fact about nature: on its own, the syntactic relation between the models gives us no reason to doubt the probabilistic independence of $X_{M}$ and $X_{A}$.

Now consider the same experiment performed in a context where the agent does believe in a universality argument for Hawking radiation, as characterised in the last section. The universality arguments give a physical justification for interpreting the syntactic isomorphism as indicative of a deeper fact about nature. In particular, they directly imply an inferential link between certain background assumptions of both the model of the source system and the model of the target system. That is, while we expect the majority of the background assumptions to remain independent, the universality argument provides a link between some background assumptions. In this sense evidence in favour of $\mathcal{A}$ may support a subset of the background assumptions $X_{M}$.

The inductive support that such an experiment would provide for $M$ in such a context will depend on how certain we are about the adequacy of the relevant background assumptions. If we already have strong independent grounds on which to assign high probabilities to the the relevant background assumptions, then there is not much added in terms of inductive support. In the context of simulating Hawking radiation via 'dumb holes', the universality argument provides a link between the crucial background assumption that in each case the effect is independent of influences of the higher energy theories. Due to the trans-Planckian problem, this independence claim is (arguably) the least well supported of the background assumptions. There is thus a clear route for strong inductive support.

In order to make explicit calculation tractable we will subsume both the universality arguments and the common background assumptions within a single variable $X$. The binary value $X$ has the values:

X: universality arguments in support of common background assumptions hold.

$\neg \mathrm{X}$ : universality arguments in support of common background assumptions do not 


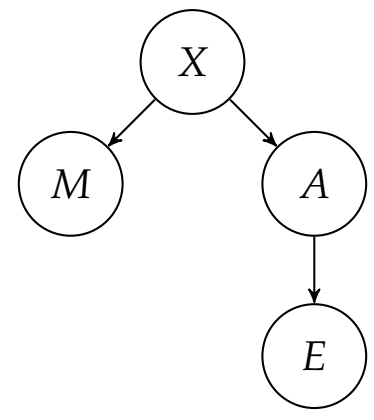

Figure 2: Simplified network for one source system with universality arguments.

hold.

So $X$ expresses a rather general claim, which can plausibly be assumed to be uncertain. If we were certain about $X$, the inference from $\mathcal{A}$ to $\mathcal{M}$ would be blocked. We will say more about this later. We will also subsume the remaining background assumptions, that is those that are not addressed by $X$, under the nodes $M$ and $A$.

Under the conditions of our assumptions, the simplified Bayesian network given in Figure 2 will then adequately model the chain of inferences involved in analogue simulation supported by universality arguments. We would like to show that $E$ confirms $M$ within a Bayesian theory of confirmation. This requires that one proves that $P(\mathrm{M} \mid \mathrm{E})>P(\mathrm{M})$. For this purpose we need to specify all prior probabilities of the 'parent node' in the Bayesian network (i.e., $X$ ) and the conditional probabilities for the other 'child nodes', given the values of their parents.

Let us simplify our notation by using the following shorthand:

$$
\begin{array}{rl}
P(\mathrm{X})=: x & P(\mathrm{M} \mid \mathrm{X})=: m_{x} \\
P(\mathrm{~A} \mid \mathrm{X})=: a_{x} & P(\mathrm{E} \mid \mathrm{A})=: e_{a} .
\end{array}
$$

The probabilities of the corresponding negated propositions are denoted with a bar, viz. $P(\mathrm{~A} \mid \overline{\mathrm{X}})=: a_{\bar{x}}, P(\overline{\mathrm{A}} \mid \mathrm{X})=: \bar{a}_{x}$ and $P(\overline{\mathrm{A}} \mid \overline{\mathrm{X}})=: \bar{a}_{\bar{x}}$.

The first central assumption is that the prior probability of $X$ lies in the open interval $(0,1)$, however, as we discussed in Sect. 3, we have theoretical arguments, in particular the result by Unruh and Schützhold, in favour of X. So a rational agent would assign:

$$
1 / 2<x<1 .
$$

The conditional probabilities are then constrained by the following conditions:

$$
\begin{aligned}
m_{x} & >m_{\bar{x}} \\
a_{x} & >a_{\bar{x}} \\
e_{a} & >e_{\bar{a}} .
\end{aligned}
$$

The conditions (2) to (4) encode probabilistically the relevant elements of the universality arguments since they allow for the possibility of a background assumption that supports both $\mathrm{M}$ and $\mathrm{A}$. The statement (5) encodes probabilistically that the empirical evidence actually plays the role of evidence in favour of the model A.

With this, the following theorem holds (the proof is in Appendix A):

Theorem 1: $P(\mathrm{M} \mid \mathrm{E})>P(\mathrm{M})$, if the conditions (3), (4) and (5) are satisfied. 
The satisfaction of Theorem 1 implies that $\mathrm{E}$ confirms $\mathrm{M}$ within a Bayesian analysis of confirmation. Note that condition (2) is not required to prove Theorem 1.

Within the framework of analogue simulation, provided we have universality arguments with prior probability that is neither unity or zero, confirmation of a hypothesis regarding the target system can obtain based upon evidence relating to the source system. It is important to note again, that having independent grounds on which to support one of the common background assumptions will 'block' the inductive support $\mathrm{E}$ can give for $\mathrm{M}$ as that background assumption already has a large marginal probability. This does not pose a problem for this account but offers a way to distinguish between those circumstances in which the novel empirical evidence $\mathrm{E}$ can provide substantial inductive support for $M$ and those circumstances it cannot be used for that purpose.

An important implication of the Bayesian analysis relates to the role of the syntactic isomorphism. The structure of the Bayesian network is such that the syntactic isomorphism is not explicitly represented. Furthermore, based upon the network, even if no syntactic isomorphism obtains between the modelling frameworks $\mathcal{M}$ and $\mathcal{A}$, one could sensibly talk about confirmation of $\mathrm{M}$ by $\mathrm{E}$, provided there exists some non-empty set of shared background assumptions. The key point is that in such circumstances although confirmation of $\mathrm{M}$ would indeed obtain, there would be no 'analogue simulation'. As discussed above, the role of the isomorphism is to guarantee that there will be a term within the modelling language of $\mathcal{M}$ that is counterpart to the term within $\mathcal{A}$ that refers to $\mathcal{E}$. Without such a term within $\mathcal{M}$ there would be no sense in which $\mathcal{S}$ is acting as a simulator for the behaviour of $\mathcal{T}$. Although the syntactic isomorphism is not explicitly represented in the network, it is implicitly stipulated within the universality argument. Furthermore, as noted above, the universality argument is exactly the reason we take the syntactic isomorphism to be indicative of a deeper fact about nature. Although it cannot be used to establish confirmation, the syntactic isomorphism is a crucial, if ultimately non-fundamental, heuristic for finding the universality argument that can.

To recapitulate, in this section we have demonstrated that confirmation via analogue simulation obtains within a Bayesian analysis provided there exists an inferential connection between the conditions of applicability of the target and system models. That is, if there exists a binary variable that is assumed to be positively correlated with the empirical adequacy of both the source and target models, then evidence in favour of the model of the source system can be used to make inferences about the target system. This, in-and-of-itself, is not a particularly surprising result, and certainly the demonstration of such in principle inferential relations is not a validation of the framework for analogue simulation that is being proposed. Rather, we take the results of this section to: i) demonstrate the internal consistency of the informal arguments towards confirmation via analogue simulation given in (Dardashti et al. 2017); and ii) provide a powerful evaluative and heuristic tool for the analysis of analogue simulation as it exists within contemporary scientific practice. Two natural directions of further development are: i) the identification and evaluation of potential cases of confirmation via analogue simulation in other scientific examples; and ii) the refinement of the Bayesian model to include cases within more than one analogue system. The second of these will be pursued in the following section. 


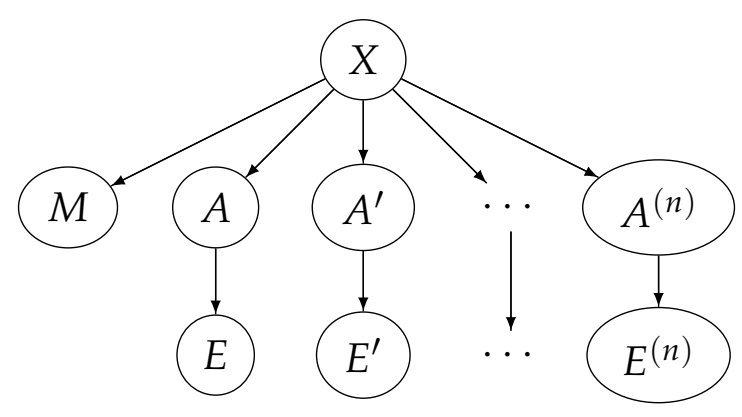

Figure 3: n-source system.

\subsection{Multiple Source Confirmation}

One important application of analogue simulation is in the context of universality arguments. In such cases the source system is 'multiply realisable' in that there are various different physical systems that can be used to implement the analogue simulation. Such a notion of 'multiply realisability' is intended to be something more than the variation of the material constitution of the source system. Such variation would involve keeping fixed the 'nomological behaviour' of the source system but changing the material constitution. Rather, the situation we are considering is when one varies the modelling frameworks used to construct the analogy, and in doing so considers equations that are syntactically isomorphic but extensionally distinct.

For example, consider again the dumb hole case. Rather than making use of the syntactic isomorphism between BEC and gravitational models we can draw inferences based upon analogue black holes constructed out of traveling refractive index interfaces in nonlinear optical media or 'slow light' in moving media (Carusotto et al. 2008). This is to vary both the material constitution and the nomological behaviour of the analogue system.

With such examples in mind, we can extend the analysis of the previous section to consider the case when we have multiple sources each providing independent evidence for the target system modelling framework. The expectation would be that adding more source systems should increase the degree of confirmation, but that this increase will eventually reach some 'saturation point'. This matches the intuition that, given some non-zero (or one) prior probability for the truth of the universality arguments, a small set of different successful realisations of the source system would be enough to provide strong evidence in favour of a hypotheses of regarding analogue behaviour in the target system.

Consider a Bayesian network for an $n$-source system (Figure 3). The question we would like to answer is how does the confirmation measure change as one increases the number of different analogue systems providing us with evidence. Following the same line of reasoning as the last section we assume:

$$
\begin{array}{cl}
a_{x}^{\prime}>a_{\bar{x}}^{\prime}, & e_{a^{\prime}}^{\prime}>e_{\bar{a}^{\prime} .}^{\prime} . \\
a_{x}^{\prime \prime}>a_{\bar{x}}^{\prime \prime} & , e_{a^{\prime \prime}}^{\prime \prime}>e_{\bar{a}^{\prime \prime}}^{\prime \prime} . \\
& \vdots \\
a_{x}^{(n)}>a_{\bar{x}}^{(n)} & , e_{a^{(n)}}^{(n)}>e_{\bar{a}^{(n)}}^{(n)} .
\end{array}
$$


We can now calculate the difference measure of confirmation, which is defined as

$$
\Delta^{(n)}:=P\left(\mathrm{M} \mid \mathrm{E}, \mathrm{E}^{\prime}, \ldots, \mathrm{E}^{(n)}\right)-P(\mathrm{M}),
$$

and obtain $\Delta^{(n)}>0$ (the proof is in Appendix B).

It can further be shown that (see Appendix B):

Theorem 2: $\Delta^{(n)}$ is a strictly increasing function of the number of source systems.

This theorem implies that as the number of different analogue systems providing evidence increases so does the degree of confirmation. ${ }^{13}$ Again, this is not a particularly surprising result. Given that confirmation via analogue simulation obtains for a single source system, one would expect that adding in more and more (independent) source systems would allow one to increase the degree of confirmation. The feature that is most interesting is not the fact that $\Delta^{(n)}$ is strictly increasing, but rather the functional form of this increase. In particular, the natural intuition is that as the number of source systems increases the increase in the degree of confirmation would eventually saturate. One of the chief virtues of the Bayesian model for analogue simulation with multiple source systems is that it allows us to give an analytical expression for such a saturation point.

First, let us consider how $\Delta^{(n)}$ changes in the large $n$ limit. A little analytical work (again see Appendix B) allows us to show that

$$
\lim _{n \rightarrow+\infty} \Delta^{(n)} \rightarrow \bar{x}\left(m_{x}-m_{\bar{x}}\right)=N_{\text {sat. }} .
$$

This means that the maximum amount of confirmation one can obtain by adding in more and more sources is bounded by some finite number, $N_{\text {sat., }}$ determined by the prior probabilities $\bar{x}, m_{x}$ and $m_{\bar{x}}$. Beyond this point, there is vanishingly small added value (in terms of confirmation) achieved by adding in more source systems. Two features of $N_{\text {sat. }}$ are worth remarking on. First, the higher the prior probability of $X$ the lower the saturation point will be. This makes sense because the more sure we are of $X$ to start with, the lower the limit on the extra information we can learn from $E, E^{\prime}, \ldots, E^{(n)}$. Second, the higher the relative likelihood of $M$ given $X$ to $M$ given $\neg$ X (i.e. $m_{x}-m_{\bar{x}}$ ), the higher the saturation point. This makes sense because the stronger the relationship between $X$ and $M$ the more we can potentially learn from $\mathrm{E}, \mathrm{E}^{\prime}, \ldots, \mathrm{E}^{(n)}$.

A further interesting feature that we can examine is the speed with which the saturation point is approached. We can examine this 'rate of saturation' by plotting $\Delta^{(n)}$ for a set of prior probabilities of $X .{ }^{14}$ As can be seen from Figure 4, the higher the prior probability of $X$, the quicker the saturation point is reached. Strikingly, for the values of the parameters considered, we find that given a prior of greater than 0.5 for $\mathrm{X}$, saturation can be reached after only three or four successful analogue experiments.

\footnotetext{
${ }^{13}$ Theorem 2 does not depend on the choice of this particular confirmation measure and will also hold if we move to another confirmation measure (Fitelson 1999).

${ }^{14}$ See Equation (23) of Appendix B. Here we have assumed for simplicity that $\gamma^{(k)}=c$ for all $k$ with $c>1 . c$ measures both the likelihood of $\mathrm{A}^{(k)}$ given $\mathrm{X}$ and the likelihood of $\mathrm{E}^{(k)}$ given $\mathrm{A}^{(k)}$. The stronger the dependence of these the stronger the exponential increase of $\Delta^{(n)}$ with $n$.
} 


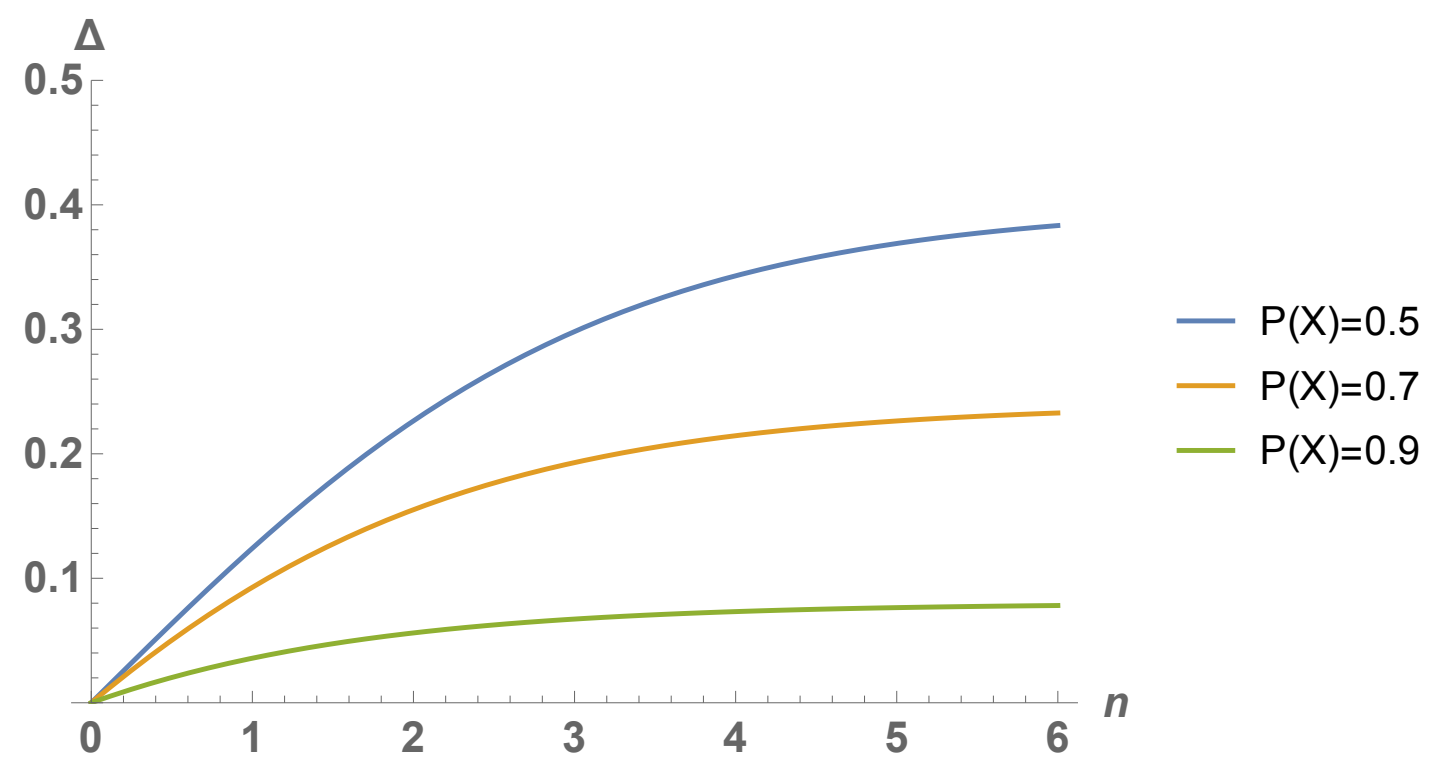

Figure 4: Confirmation measure dependence and saturation point.

This result is in tune with scientific intuitions regarding analogue simulation in the context of universality arguments. Consider, in particular the dumb hole Hawking radiation case. There has been, thus far, only one implementation of a source system that is reported to display the quantum Hawking effect: the Bose-Einstein condensate experiments of (Steinhauer 2016). ${ }^{15}$ Given initial confidence in the universality arguments, if another different implementation of a source system displaying the Hawking effect was achieved, that should surely radically increase the belief in the astrophysical Hawking effect. However, once a few such examples were constructed, one would quickly stop gaining new insight. Conversely, given initial skepticism regarding the universality arguments, a second implementation of the dumb hole source system would not radically increase the belief in the astrophysical Hawking effect. Furthermore, in such circumstances it would only be after a diverse and extensive range of implementations of source systems that one would stop believing that new examples gave new information.

\subsection{Confirmation Dependence on Source System}

Although most of the proposed analogue models of black hole Hawking radiation have not been tested yet, there can be significant differences in our prior belief regarding the adequacy of these models. One reason for this is that the modelling may rely on a strong theoretical basis in one system but a rather conjectural basis in the other. Another reason is that one may have good control of the experimental setup, such that one has more reason to rely on the adequate realization of the various idealizing assumptions involved in the derivation of the model. This will have as an effect that we would assign different marginal probabilities to the analogue models. A question thus arises, namely how do the variations in the marginal probabilities

\footnotetext{
${ }^{15}$ We should note again that the earlier experiments of (Weinfurtner et al. 2013) show classical aspects of Hawking radiation in an analogue system using surface waves. Modelling the inferential relationship between the classical and quantum experiments is an interesting open question.
} 
one assigns to the adequacy of the analogue model affect the confirmation behaviour of the analogue setup.

For simplicity, we will only consider the variation for the one-source model (Fig. 2). One can now show (see App. $C$ for details and the proof):

Theorem 3: $\partial \Delta / \partial a<0$, if conditions (2) to (5) hold.

Here the derivative is taken with respect to $a:=P(\mathrm{~A})$.

This plausible result implies that an assignment of a higher probability to the adequacy of the analogue model will have the effect of a decrease in the confirmation of the adequacy of the target model by the observation of the analogue Hawking effect. Or to put it differently: the more certain we are about the adequacy of the model we are experimenting on, the less effective is the evidence obtained there. Significantly, this result has direct implications for the respective epistemic value of future analogue experiments. In particular, all else being equal, it implies that scientists will learn more by conducting future analogue experiments using media about which we are less certain regarding the fundamental physics, than those using media about which we are more confident.

\section{Conclusion and Prospectus}

History is replete with examples of 'transformative' technology having a profound and lasting impact on the methodological foundations of science. Much recent literature in the philosophy of science has focused on the sense in which computer simulation should be taken to have had such an impact. ${ }^{16}$ Analogue simulation is a new inferential tool found at the cutting edge of modern science that we see good reasons to take as potentially transformative. Building upon the initial analysis of (Dardashti et al. 2017), in this paper we have applied a Bayesian analysis to explicate the structure of inferences that analogue simulation can and cannot allow us to make.

Our three principal results are: i) that 'single source' confirmation via analogue simulation can obtain under certain conditions; ii) that 'multiple source' confirmation via analogue simulation displays the generic feature of saturation in confirmatory power; iii) analogue experiments in which we are more confident about the fundamental physics provide less confirmation than those about which we are more confident. Our results provide a basis to both formalise the epistemic value of analogue experiments that have been conducted and to advise scientists as to the respective epistemic value of future analogue experiments.

\section{A Proof of Theorem 1}

We consider the Bayesian network depicted in Figure 1 and have to show that

$$
P(\mathrm{M} \mid \mathrm{E})=\frac{P(\mathrm{M}, \mathrm{E})}{P(\mathrm{E})}>P(\mathrm{M})
$$

\footnotetext{
${ }^{16}$ See for example (Humphreys 1995; Humphreys 2004; Hartmann 1996; Winsberg 1999; Winsberg 2010; Parker 2009).
} 
The joint probability can be obtained in the following way: ${ }^{17}$

$$
\begin{aligned}
P(\mathrm{M}, \mathrm{E}) & =\sum_{X, A} P(X, \mathrm{M}, A, \mathrm{E}) \\
& =\sum_{X, A} P(X) P(\mathrm{M} \mid X) P(A \mid X) P(\mathrm{E} \mid A) \\
& =x m_{x}\left(a_{x} e_{a}+\bar{a}_{x} e_{\bar{a}}\right)+\bar{x} m_{\bar{x}}\left(a_{\bar{x}} e_{a}+\bar{a}_{\bar{x}} e_{\bar{a}}\right) \\
& =x m_{x} \alpha+\bar{x} m_{\bar{x}} \beta
\end{aligned}
$$

where we have defined

$$
\begin{aligned}
\alpha & :=a_{x} e_{a}+\bar{a}_{x} e_{\bar{a}} \\
\beta & :=a_{\bar{x}} e_{a}+\bar{a}_{\bar{x}} e_{\bar{a}} .
\end{aligned}
$$

Similarly we obtain

$$
\begin{aligned}
P(\mathrm{E}) & =\sum_{X, A, M} P(X, M, A, \mathrm{E}) \\
& =x \alpha+\bar{x} \beta
\end{aligned}
$$

and

$$
P(\mathrm{M})=x m_{x}+\bar{x} m_{\bar{x}} .
$$

Defining the difference measure $\Delta:=P(\mathrm{M} \mid \mathrm{E})-P(\mathrm{M})$, we need to show that $\Delta$ is larger than zero. After some algebraic manipulation one obtains

$$
\begin{aligned}
\Delta & =\frac{x m_{x} \alpha+\bar{x} m_{\bar{x}} \beta-\left(x m_{x}+\bar{x} m_{\bar{x}}\right)(x \alpha+\bar{x} \beta)}{x \alpha+\bar{x} \beta} \\
& =\frac{\bar{x} x\left(m_{x}-m_{\bar{x}}\right)(\alpha-\beta)}{x \alpha+\bar{x} \beta} .
\end{aligned}
$$

It is easy to show that

$$
\alpha-\beta=\left(a_{x}-a_{\bar{x}}\right)\left(e_{a}-e_{\bar{a}}\right)
$$

Hence it follows that

$$
\Delta=\frac{\bar{x} x\left(m_{x}-m_{\bar{x}}\right)\left(a_{x}-a_{\bar{x}}\right)\left(e_{a}-e_{\bar{a}}\right)}{x \alpha+\bar{x} \beta} .
$$

So if (3), (4) and (5) are satisfied it follows that $\Delta>0$, which needed to be shown to proof Theorem 1.

\section{B Proofs for $n$ Source Systems}

To see how the previous theorem can be generalized to the $n$ source systems represented in Figure 3 let us consider first the 2-source system represented in Figure 5.

\footnotetext{
${ }^{17}$ See (Bovens and Hartmann 2004, Sect. 3.5) on the general methodology of reading joint probabilities from Bayesian networks.
} 


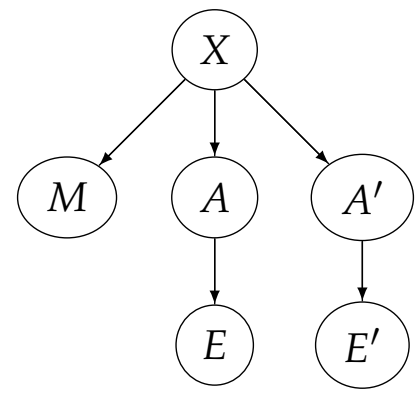

Figure 5: 2-source system.

We need to show that $P\left(\mathrm{M} \mid \mathrm{E}, \mathrm{E}^{\prime}\right)=P\left(\mathrm{M}, \mathrm{E}, \mathrm{E}^{\prime}\right) / P\left(\mathrm{E}, \mathrm{E}^{\prime}\right)>P(\mathrm{M})$. Let us start with the following joint probability

$$
\begin{aligned}
P\left(\mathrm{M}, \mathrm{E}, \mathrm{E}^{\prime}\right) & =\sum_{X, A, A^{\prime}} P\left(X, \mathrm{M}, A, \mathrm{E}, A^{\prime}, \mathrm{E}^{\prime}\right) \\
& =\sum_{X, \mathrm{~A}_{,} \mathrm{A}^{\prime}} P(\mathrm{X}) P(\mathrm{M} \mid \mathrm{X}) P(\mathrm{~A} \mid \mathrm{X}) P(\mathrm{E} \mid \mathrm{A}) P\left(\mathrm{~A}^{\prime} \mid \mathrm{X}\right) P\left(\mathrm{E}^{\prime} \mid \mathrm{A}^{\prime}\right) \\
& =x m_{x} \alpha \alpha^{\prime}+\bar{x} m_{\bar{x}} \beta \beta^{\prime},
\end{aligned}
$$

where $\alpha^{\prime}$ and $\beta^{\prime}$ is defined identically to (13) and (14) with $e$ and $a$ replaced with $e^{\prime}$ and $a^{\prime}$.

Similarly we obtain

$$
\begin{aligned}
P\left(\mathrm{E}, \mathrm{E}^{\prime}\right) & =\sum_{X, A, A^{\prime}} P(X) P(\mathrm{E} \mid A) P(A \mid X) P\left(\mathrm{E}^{\prime} \mid A^{\prime}\right) P\left(A^{\prime} \mid X\right) \\
& =x \alpha \alpha^{\prime}+\bar{x} \beta \beta^{\prime} .
\end{aligned}
$$

Defining $\Delta^{\prime}:=P\left(\mathrm{M} \mid \mathrm{E}, \mathrm{E}^{\prime}\right)-P(\mathrm{M})$ it follows that

$$
\Delta^{\prime}=\frac{x \bar{x}\left(m_{x}-m_{\bar{x}}\right)\left(\alpha \alpha^{\prime}-\beta \beta^{\prime}\right)}{x \alpha \alpha^{\prime}+\bar{x} \beta \beta^{\prime}} .
$$

Now $\alpha \alpha^{\prime}-\beta \beta^{\prime}>0$ iff $(\alpha / \beta) \cdot\left(\alpha^{\prime} \beta^{\prime}\right)>1$. This holds if $\alpha>\beta$ and $\alpha^{\prime}>\beta^{\prime}$. Both conditions hold because of assumptions (4) to (6). Hence, $\Delta^{\prime}$ is greater than zero.

It is straightforward to generalise our discussion to the $n$-source system represented in Figure 3. Here we need to show that $\Delta^{(n)}:=P\left(\mathrm{M} \mid \mathrm{E}, \mathrm{E}^{\prime}, \ldots, \mathrm{E}^{(n)}\right)-P(\mathrm{M})>$ 0 . It follows from the above consideration that

$$
\Delta^{(n)}=\frac{x \bar{x}\left(m_{x}-m_{\bar{x}}\right)\left(\prod_{k=0}^{n} \alpha^{(k)}-\prod_{k=0}^{n} \beta^{(k)}\right)}{x \prod_{k=0}^{n} \alpha^{(k)}+\bar{x} \prod_{k=0}^{n} \beta^{(k)}},
$$

with $\alpha^{(0)}=\alpha$ and $\beta^{(0)}=\beta$. We have again $\Delta^{(n)}>0$ once (4) to (8) are satisfied.

Let us define $\gamma^{(n)}:=\prod_{k=0}^{n} \alpha^{(k)} / \beta^{(k)}$. Since $\alpha^{(k)}>\beta^{(k)}$ for all $k, \gamma^{(n)}$ increases as $n$ increases. Furthermore, we obtain

$$
\frac{\partial \Delta^{(n)}}{\partial \gamma^{(n)}}=\frac{x \bar{x}\left(m_{x}-m_{\bar{x}}\right)}{\left(x \gamma^{(n)}+\bar{x}\right)^{2}}>0
$$


We conclude that the amount of confirmation increases as $n$ increases, i.e. as the number of analogue systems providing evidence increases. Setting $\kappa:=x \bar{x}\left(m_{x}-m_{\bar{x}}\right)$ we obtain for the large $n$ behaviour:

$$
\lim _{n \rightarrow+\infty} \Delta^{(n)}=\lim _{x \rightarrow+\infty} \kappa \frac{\gamma^{(n)}-1}{x \gamma^{(n)}+\bar{x}} \rightarrow \frac{\kappa}{x} .
$$

This completes the proof of Theorem 2.

\section{Proof of Theorem 3}

We need to show how the confirmation measure (19) varies as our belief regarding the adequacy of the analogue model varies. That is, we need to calculate

$$
\begin{aligned}
\frac{\partial \Delta}{\partial a} & =\frac{\partial a_{x}}{\partial a} \frac{\partial \Delta}{\partial a_{x}}+\frac{\partial a_{\bar{x}}}{\partial a} \frac{\partial \Delta}{\partial a_{\bar{x}}}+\frac{\partial x}{\partial a} \frac{\partial \Delta}{\partial x} \\
& =\frac{1}{x} \frac{\partial \Delta}{\partial a_{x}}+\frac{1}{\bar{x}} \frac{\partial \Delta}{\partial a_{\bar{x}}}+\frac{1}{\left(a_{x}-a_{\bar{x}}\right)} \frac{\partial \Delta}{\partial x}
\end{aligned}
$$

where we have used $a:=x a_{x}+\bar{x} a_{\bar{x}}$ in the last step. Taking the derivatives yields

$$
\begin{aligned}
\frac{\partial \Delta}{\partial a_{x}} & =\frac{k x \bar{x}}{(x \alpha+\bar{x} \beta)^{2}}\left(x \alpha+\bar{x} \beta-\left(a_{x}-a_{\bar{x}}\right)\left(e_{a}-e_{\bar{a}}\right) x\right), \\
\frac{\partial \Delta}{\partial a_{\bar{x}}} & =-\frac{k x \bar{x}}{(x \alpha+\bar{x} \beta)^{2}}\left(x \alpha+\bar{x} \beta+\left(a_{x}-a_{\bar{x}}\right)\left(e_{a}-e_{\bar{a}}\right) \bar{x}\right), \\
\frac{\partial \Delta}{\partial x} & =\frac{k\left(a_{x}-a_{\bar{x}}\right)}{(x \alpha+\bar{x} \beta)^{2}}(x \alpha+\bar{x} \beta-x(1+x) \alpha-x \bar{x} \beta) .
\end{aligned}
$$

Here we have used the shorthand $k:=\left(m_{x}-m_{\bar{x}}\right)\left(e_{a}-e_{\bar{a}}\right)$. Plugging these expressions into eq. (26) one obtains after some manipulation that

$$
\frac{\partial \Delta}{\partial a}=\frac{k}{(x \alpha+\bar{x} \beta)^{2}}(\bar{x}(1+\bar{x}) \beta-x(1+x) \alpha) .
$$

Assuming that (3) and (5) hold makes $k$ positive and assuming that (2), (4) and (5) hold makes the large bracket in (27) negative, i.e. it leads to $\partial \Delta / \partial a<0$. This completes the proof of Theorem 3.

\section{References}

Anderson, M., J. Ensher, M. Matthews, C. Wieman, and E. Cornell (1995). 'Observation of Bose-Einstein condensation in a dilute atomic vaporin a dilute atomic vapor'. Science 269, 14.

Bailer-Jones, D. M. (2009). Scientific Models in Philosophy of Science. Pittsburgh: University of Pittsburgh Press.

Barceló, C., L. J. Garay, and G. Jannes (2009). 'Sensitivity of Hawking radiation to superluminal dispersion relations'. Physical Review D 79(2), 024016. 
Bartha, P. (2013). 'Analogy and analogical reasoning'. In: E. N. Zalta (ed.), The Stanford Encyclopedia of Philosophy (Fall 2013 ed.).

Bartha, P. F. (2010). By Parallel Reasoning: The Construction and Evaluation of Analogical Arguments. Oxford: Oxford University Press.

Batterman, R. W. (2000). 'Multiple realizability and universality'. The British Journal for the Philosophy of Science 51(1), 115-145.

Belgiorno, F., S. Cacciatori, M. Clerici, V. Gorini, G. Ortenzi, L. Rizzi, E. Rubino, V. Sala, and D. Faccio (2010). 'Hawking radiation from ultrashort laser pulse filaments'. Physical Review Letters 105(20), 203901.

Betz, G. (2013). 'Revamping hypothetico-deductivism: A dialectic account of confirmation'. Erkenntnis 78(5), 991-1009.

Bose, S. N. (1924). 'Plancks Gesetz und Lichtquantenhypothese'. Zeitschrift für Physik 26(3), 178.

Bovens, L. and S. Hartmann (2004). Bayesian Epistemology. Oxford: Oxford University Press.

Carusotto, I., S. Fagnocchi, A. Recati, R. Balbinot, and A. Fabbri (2008). 'Numerical observation of Hawking radiation from acoustic black holes in atomic BoseEinstein condensates'. New Journal of Physics 10(10), 103001.

Corley, S. (1998). 'Computing the spectrum of black hole radiation in the presence of high frequency dispersion: An analytical approach'. Physical Review D 57(10), 6280.

Coutant, A., R. Parentani, and S. Finazzi (2012). 'Black hole radiation with short distance dispersion, an analytical S-matrix approach'. Physical Review D 85(2), 024021.

Dardashti, R., K. P. Thébault, and E. Winsberg (2017). 'Confirmation via analogue simulation: what dumb holes could tell us about gravity'. British Journal for the Philosophy of Science 68(1), 55-89.

Dobson, D. (2016). 'Geophysics: Earth's core problem'. Nature 53(7605), 45-45.

Einstein, A. (1924). 'Quantentheorie des einatomigen idealen Gases'. Sitzungsberichte der Preussische Akadmie der Wissenschaften, 261-267.

Euvé, L.P., S. Robertson, N. James, A. Fabbri, and G. Rousseaux (2018) Scattering of surface waves on an analogue black hole arxiv.org/pdf/1806.05539

Fitelson, B. (1999). 'The plurality of Bayesian measures of confirmation and the problem of measure sensitivity'. Philosophy of Science 66, 362-378.

Franklin, A. (1989). The Neglect of Experiment. Cambridge: Cambridge University Press.

Franklin, A. and S. Perovic (2016). 'Experiment in physics'. In E. N. Zalta (Ed.), The Stanford Encyclopedia of Philosophy (Winter 2016 Edition).

Garay, L., J. Anglin, J. Cirac, and P. Zoller (2000). 'Sonic analog of gravitational black holes in Bose-Einstein condensates'. Physical Review Letters 85(22), 4643.

Giddings, S. B. (2016). 'Hawking radiation, the Stefan-Boltzmann law, and unitarization'. Physics Letters B 754, 39-42. 
Glymour, C. (1980). Theory and Evidence. Princeton: Princeton University Press.

Gryb, S., P. Palacios, and K. P. Y. Thébault (2018). ‘On the Universality of Hawking Radiation' (in preparation).

Harlow, D. (2016). 'Jerusalem lectures on black holes and quantum information'. Reviews of Modern Physics 88(1), 015002.

Hartmann, S. (1996). 'The world as a process'. In R. Hegselmann et al. (eds.), Modelling and Simulation in the Social Sciences from the Philosophy of Science Point of View. Dordrecht: Springer, pp. 77-100.

Hartmann, S. and J. Sprenger (2010). 'Bayesian epistemology'. In S. Bernecker and D. Pritchard (eds.), Routledge Companion to Epistemology. London: Routledge, pp. 609-620.

Hawking, S. W. (1975). 'Particle creation by black holes'. Communications in Mathematical Physics 43(3), 199-220.

Helfer, A. D. (2010). 'Comment on "Insensitivity of Hawking radiation to an invariant planck-scale cutoff"'. Physical Review D 81(10), 108501.

Hempel, C. G. (1965). Aspects of Scientific Explanation and other Essays in the Philosophy of Science. New York: Free Press.

Hesse, M. (1964). 'Analogy and confirmation theory'. Philosophy of Science 31(4), 319-327.

Hesse, M. B. (1963). Models and Analogies in Science. London: Sheed and Ward.

Hesse, M. B. (1974). The Structure of Scientific Inference. Berkeley: University of California Press.

Himemoto, Y. and T. Tanaka (2000). 'Generalization of the model of Hawking radiation with modified high frequency dispersion relation'. Physical Review D 61(6), 064004.

Hume, D. (1738/1978). A Treatise of Human Nature. Oxford: Oxford University Press.

Humphreys, P. (1995). 'Computational science and scientific method'. Minds and Machines 5(4), 499-512.

Humphreys, P. (2004). Extending Ourselves: Computational Science, Empiricism, and Scientific Method. Oxford: Oxford University Press.

Jacquet, M. (2018) Negative Frequency at the Horizon. Springer.

Jacobson, T. (1996). 'Introductory lectures on black hole thermodynamics'. ¡www.physics.umd.edu/grt/taj/776b/lectures.pdfi.

Keynes, J. M. (1921). A Treatise on Probability. London: Macmillan \& Co.

Konôpková, Z., R. S. McWilliams, N. Gómez-Pérez, and A. F. Goncharov (2016). 'Direct measurement of thermal conductivity in solid iron at planetary core conditions'. Nature 534(7605), 99-101.

Leonhardt, U. and T. G. Philbin (2008). 'The case for artificial black holes'. Philosophical Transactions of the Royal Society A: Mathematical, Physical and Engineering Sciences 366(1877), 2851-2857.

Liberati, S., A. Prain, and M. Visser (2012). 'Quantum vacuum radiation in optical glass'. Physical Review D 85(8), 084014. 
Nguyen, H., D. Gerace, I. Carusotto, D. Sanvitto, E. Galopin, A. Lemaître, I. Sagnes, J. Bloch, and A. Amo (2015). 'Acoustic black hole in a stationary hydrodynamic flow of microcavity polaritons'. Physical Review Letters 114(3), 036402.

Norton, J. D. (2011). 'Analogy'. Manuscript, http://www. pitt. edu/ jdnorton/papers/material_theory/Analogy. pdf.

Parker, W. S. (2009). 'Does matter really matter? Computer simulations, experiments, and materiality'. Synthese 169(3), 483-496.

Philbin, T. G., C. Kuklewicz, S. Robertson, S. Hill, F. König, and U. Leonhardt (2008). 'Fiber-optical analog of the event horizon'. Science 319(5868), 1367-1370.

Polchinski, J. (1995). 'String theory and black hole complementarity'. arXiv preprint hep-th/9507094.

Reid, T. and W. Hamilton (1850). Essays on the Intellectual Powers of Man. Cambridge: J. Bartlett.

Salmon, W. (1967). The Foundations of Scientific Inference. Pittsburgh: University of Pittsburgh Press.

Salmon, W. (1990). 'Rationality and objectivity in science or Tom Kuhn meets Tom Bayes'. In C. Wade Savage (ed.), Scientific Theories. Minneapolis: University of Minnesota Press, pp. 175-204.

Steinhauer, J. (2016). 'Observation of quantum Hawking radiation and its entanglement in an analogue black hole'. Nature Physics (Advance Online Publication).

Thébault, K. P. Y. (2016). 'What can we learn from analogue experiments?' (in preparation).

Thompson, R. and L. Ford (2008). 'Enhanced black hole horizon fluctuations'. Physical Review D 78(2), 024014.

Unruh, W. (1981). 'Experimental black-hole evaporation?'. Physical Review Letters 46(21), 1351-1353.

Unruh, W. and R. Schützhold (2012). 'Hawking radiation from "phase horizons" in laser filaments?'. Physical Review D 86(6), 064006.

Unruh, W. G. (1976). 'Notes on black-hole evaporation'. Physical Review D 14(4), 870.

Unruh, W. G. (2014). 'Has Hawking radiation been measured?'. Foundations of Physics 44(5), 532-545.

Unruh, W. G. and R. Schützhold (2005). 'Universality of the Hawking effect'. Physical Review D 71(2), 024028.

Wallace, D. (2017). 'The case for black hole thermodynamics, part I: phenomenological thermodynamics'. https://arxiv.org/abs/1710.02724.

Weinfurtner, S., E. W. Tedford, M. C. Penrice, W. G. Unruh, and G. A. Lawrenc (2013). 'Classical aspects of Hawking radiation verified in analogue gravity experiment'. In: D. Faccio et al. (eds.), Analogue Gravity Phenomenology, Vol. 870 of Lecture Notes in Physics. Dordrecht: Springer, pp. 167-180.

Winsberg, E. (1999). 'Sanctioning models: The epistemology of simulation'. Science in Context 12(2), 275-292. 
Winsberg, E. (2010). Science in the Age of Computer Simulation. Chicago: University of Chicago Press.

Wüthrich, C. (2017). 'Are black holes about information?'. arXiv preprint arXiv:1708.05631. 